\title{
Simulación Mediante Elemento Finito de Sistemas Intramedulares Telescópicos para Rehabilitación de Pacientes con Osteogénesis Imperfecta
}

\section{Simulation by Finite Element Method of Intramedullary Telescopic Systems for Rehabilitation of Patients with Osteogenesis Imperfecta}

\author{
J. L. Rueda-Arreguín', C. R. Torres-San Miguel', V. Ramírez-Vela', G. Urriolagoitia-Sosa', L. Martínez-Sáez² \\ Instituto Politécnico Nacional \\ ¿Universidad Politécnica de Madrid
}

\section{RESUMEN}

En el trabajo se plantea el análisis de diferentes geometrías para un dispositivo intramedular, las cuales ayudan a reducir y evitar la migración, deformación y rotura del implante en tejido óseo afectado con Osteogénesis Imperfecta (OI). Se realizaron diseños en CAD de diferentes prototipos, donde se analizan las propiedades mecánicas en el alma del dispositivo, así como en las roscas distal y proximal de los implantes macho y hembra. Asimismo, se obtuvieron modelos 3D de huesos de un infante afectado con OI para realizar simulaciones mediante elemento finito de la interacción entre el hueso y el dispositivo intramedular. Los resultados muestran que los prototipos propuestos disminuyen la deformación del dispositivo, así como el aumento en la rigidez de la relación hueso-prótesis. Asimismo, las roscas generaron un menor esfuerzo en la unión con el hueso, lo que prevé un menor daño al tejido óseo. El trabajo se limitó al análisis numérico del rediseño de implantes telescópicos intramedulares para afectados con OI. Concluyendo que la geometría semicircular $3 / 4$ de caña, otorga un óptimo resultado en las pruebas realizadas, al tiempo que las roscas ACME proveen una mejor sujeción en las epífisis distal y proximal de los huesos largos. 


\section{ABSTRACT}

In this work we propose the analysis of different geometries for an intramedullary device, which help to reduce and avoid the migration, deformation and rupture of the implant in bone tissue affected with Osteogenesis Imperfecta (OI). Designs of different prototypes were made in CAD, where the mechanical properties in the device's soul are analyzed, as well as in the distal and proximal threads of the male and female implants. Likewise, 3D bone models of an affected infant with OI were obtained to perform finite element simulations of the interaction between the bone and the intramedullary device. The results show that the prototypes proposed decrease the strain of the device, as well as the increase in the stiffnes of the bone-prosthesis relationship. Also, the threads generated less stress in the union with the bone, which provides less damage to the bone tissue. The work was limited to the numerical analysis of the redesign of intramedullary telescopic implants for patients with OI. Concluding that the semicircular geometry $3 / 4$ of cane, gives an optimal result in the tests carried out, while the ACME threads provide a better subjection in the distal and proximal epiphyses of the long bones.

KEYWORDS: Telescopic system; Osteogenesis Imperfecta; Numerical Analysis

\section{Correspondencia}

DESTINATARIO: Chistopher René Torres San Miguel

INSTITUCIÓN: Instituto Politécnico Nacional

DIRECCIÓN: Unidad Profesional Adolfo López Mateos

"Zacatenco", Edificio 5, 2do. Piso, Col. Lindavista,

C. P. 07320, Ciudad de México, CDMX, México

CORREO ELECTRÓNICO: ctorress@ipn.mx

\section{Fecha de recepción:}

29 de junio de 2018

Fecha de aceptación:

24 de febrero 2019 


\section{INTRODUCCIÓN}

La Osteogénesis Imperfecta (OI), también llamada como la enfermedad de los huesos de cristal tiene como causa un desorden genético hereditario, que afecta principalmente la producción de colágeno, lo que trae como consecuencia en huesos con una densidad ósea y resistencia mecánica por debajo de lo normal ${ }^{[1]}$. Su tratamiento suele necesitar de la intervención de un equipo multidisciplinario; debido a las variables clínicas que se presentan en la enfermedad; dentro de las áreas más comunes se encuentran la rehabilitación física, tratamiento dental, terapia farmacológica, ortopedia e intervención quirúrgica son las que destacan ${ }^{[2]}$.

A lo largo de la historia, las fracturas en huesos largos se han tratado mediante el uso de la osteosíntesis, la cual consiste en fijar los segmentos de hueso con ayuda de elementos de sujeción, como lo son placas metálicas, dispositivos intramedulares (clavos, tornillos y alambres), agujas y pines, entre otros, dependiendo de la gravedad de la fractura. Estos métodos resultan poco eficientes para el tratamiento de la OI, debido al principio manejado para su fijación que consiste en la penetración del hueso en diferentes zonas para el ajuste del dispositivo utilizado ${ }^{[3]}$.

Posteriormente, debido a los excelentes resultados obtenidos al tratar las fracturas mediante los dispositivos intramedulares, se pensó en aplicar el mismo método en pacientes afectados con OI. Sin embargo, rápidamente se identificó el problema de estos dispositivos al tratar esta enfermedad, el cual presentaba dificultad en el teléscopado del dispositivo, es decir, no crecía junto con el hueso, lo que llegaba a ocasionar un desplazamiento del clavo dentro del hueso obligando a realizar varias cirugías para el reacomodamiento de este ${ }^{[4]}$; ejemplo de lo anterior ocurre en 1959, cuando Solfield y Millar publican los resultados de la técnica quirúrgica que empleaban en el hospital ChicagoShriners desde 1948 cuyo título es, Fragmentation, realignment and intramedullary rod fixation of deformities of the long bones in children, donde varios pacientes eran niños afectados con OI, y un elevado porcentaje de los infantes mostraban nuevas fracturas al ser tratados con clavos intramedulares ${ }^{[5]}$.

Conociendo el problema se trataron las fracturas con clavos intramedulares elásticos, Elastic Stable Intramedullary Nail, ESIN por sus siglas en inglés. Sin embargo, un nuevo problema surgió en estos clavos al ser sobre pasados por el hueso debido al crecimiento del infante, por lo que para asegurar un buen funcionamiento se optó por fijar el dispositivo en las epífisis distal y proximal del hueso ${ }^{[6]}$. En los años 60's se desarrollan los clavos telescópicos, los cuales al ser fijados en las epífisis redujeron dramáticamente el número de cirugías necesarias en un niño, no obstante, se seguían presentando complicaciones como el hecho que el crecimiento del niño sobrepasara el rango telescópico establecido para el clavo ${ }^{[7]}$.

Inicialmente el dispositivo Bailey-Dubow ${ }^{\mathrm{TM}}$ fue el clavo telescópico utilizado para el tratamiento de la OI [5], documentando buenos porcentajes de éxito, este implante consiste en la inserción de sus dos componentes por cada una de las epífisis, es decir se realizan 2 perforaciones en el hueso. Posteriormente se desarrolló el dispositivo Fassier-Duval ${ }^{\mathrm{TM}}$, el cual incorpora 2 roscas en ambos extremos del implante lo que permite ser introducido por un solo orificio, reduciendo así el número de operaciones a realizar para su inserción y extracción ${ }^{[7]}$. En la actualidad los implantes más utilizados para el tratamiento de la rehabilitación de huesos largos en pacientes con OI son los dos clavos telescópicos antes mencionados, aunque dependiendo del tipo de OI también se pueden utilizar clavos intramedulares elásticos o de fijación.

En el presente trabajo se revisarán diferentes geometrías para el diseño de un clavo telescópico especializado en pacientes con OI tipo III, para reducir los pro- 
blemas existentes y conseguir un mejor porcentaje de éxito en la rehabilitación de estos pacientes. Se llevaron a cabo análisis numéricos con base en la norma ASTM 1264 a los clavos telescópicos propuestos. Finalmente, se muestran comparaciones entre las geometrías propuestas que conllevan a prevenir migraciones en el tratamiento.

\section{METODOLOGIAA}

La metodología aplicada en este trabajo se desarrolla sobre el rediseño de un dispositivo intramedular basado en las complicaciones que se encuentran documentadas dentro de la literatura acerca del dispositivo intramedular telescópico. El diseño de este dispositivo consiste en dos elementos cilíndricos, uno de ellos tubular, el cual se denomina implante hembra, y el segundo sólido, pero con un diámetro cercano al interno del implante hembra, llamado implante macho. Cada implante posee en un extremo una sección roscada, en el implante hembra de $1.65 \mathrm{~cm}$ y en el macho de entre $1.00 \mathrm{~cm}$ y $1.5 \mathrm{~cm}$. La rosca que se emplea en el implante hembra es una rosca de perfil circular mientras que, en el implante macho el perfil es trapezoidal.

Basándose en las complicaciones encontradas dentro de la literatura, se proponen modificaciones en la geometría del implante macho con la finalidad de prevenir una rotación que desencadene en la pérdida de fijación del implante con la epífisis. Asimismo, se plantean modificaciones dentro de las roscas de los implantes, para lograr una mejor fijación entre implante-hueso y evitar la migración del dispositivo intramedular.

La metodología se desarrolla en este trabajo mediante la aplicación del método de elemento finito, en 15 diferentes casos de estudios, de los cuales 7 son con respecto a pruebas de flexión y torsión en el área del alma del dispositivo, 6 casos de estudio consisten en los análisis de la interacción de un modelo 3D de un hueso con OI y las diferentes roscas propuestas, con la finalidad de determinar la resistencia mecánica ejercido en el sistema óseo, finalmente, 2 casos de estudio del tejido óseo afectado con OI sin implante y con un dispositivo intramedular colocado.

Se propusieron diferentes secciones transversales con las que se pueden atacar los problemas de rotación del implante macho. Las geometrías propuestas para el diseño de un nuevo clavo telescópico son: polígono hexagonal, circular con pestaña y un semicírculo 3/4 de caña, como se observa en la Figura 1.

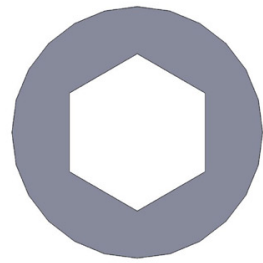

a)

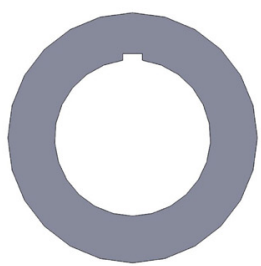

b)

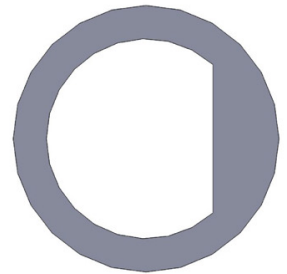

c)
FIGURA 1. Casos de estudio. a) Hexagonal.

b) Circular con pestaña. c) Semicírculo 3/4 de caña.

\section{Prueba de flexión}

Se realizó el análisis numérico en el dispositivo Fassier-Duval ${ }^{\mathrm{TM}}$ utilizando las condiciones de flexión en cuatro puntos establecidas por la norma ASTM 1264 para dispositivos intramedulares, con la finalidad de obtener un marco de referencia con el cual comparar las nuevas propuestas. Las propiedades del material empleado para el análisis numérico corresponden a un Acero Inoxidable 316L grado médico, el cual cuenta con un módulo de Young de $200 \mathrm{GPa}$, un coeficiente de Poisson de 0.268 y un límite elástico de $170 \mathrm{MPa}{ }^{[8]}$.

Dentro de la literatura se conoce la biomecánica de las fuerzas que interactúan dentro de los huesos de la pierna, por lo que, se determinan las cargas con base al peso del paciente y la curvatura del hueso, metodología que se emplea en la determinación de las cargas aplicadas al sistema propuesto del clavo intramedular [9]. En el desarrollo del trabajo, se considera un paciente de estudio que presenta OI tipo III de 6 años de edad y un peso de $11 \mathrm{~kg}$. 
Con base en lo anterior, se aplicó una carga perpendicular de $100 \mathrm{~N}$ para simular el caso más extremo del paciente, el cual refiere a una bipedestación (Figura 2).

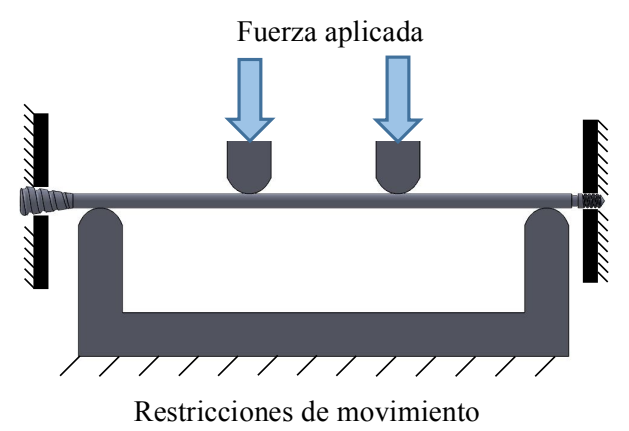

FIGURA 2. Condiciones de frontera

para prueba de flexión en 4 puntos.

A continuación, se efectúa el discretizado de las piezas dentro del análisis, para eso se realiza un mallado libre con elementos triangulares. La Tabla 1 muestra el número de elementos y nodos de los casos analizados.

TABLA 1. Número de elementos

y nodos generados para el análisis.

\begin{tabular}{|c|c|c|}
\hline $\begin{array}{c}\text { Dispositivo } \\
\text { para analizar }\end{array}$ & $\begin{array}{c}\text { No. de } \\
\text { elementos }\end{array}$ & $\begin{array}{c}\text { No.de } \\
\text { nodos }\end{array}$ \\
\hline Fassier-Duval ${ }^{\text {Nex }}$ & 24352 & 39626 \\
\hline Hexagonal & 18874 & 33633 \\
\hline $\begin{array}{c}\text { Circular } \\
\text { con pestaña }\end{array}$ & 18615 & 33669 \\
\hline $\begin{array}{c}\text { Semicírculo } \\
\text { 3/4 de caña }\end{array}$ & 16973 & 30461 \\
\hline
\end{tabular}

\section{Prueba de torsión}

Se realizaron pruebas de torsión establecidas dentro de la norma, a los 3 diseños propuestos para determinar su resistencia a la misma, y prevenir rotaciones que provoquen una deficiente rehabilitación en los huesos largos de los pacientes con OI.

Las condiciones bajo las que se someterá el estudio consistirán en aplicar un punto de sujeción en el extremo del implante externo y una fuerza de torsión de $0.6 \mathrm{~N} / \mathrm{m}$ en el extremo del implante interno. Se utiliza esta magnitud debido a que, al realizar el movimiento del tobillo, el único elemento que genera resistencia es el peso del pie, el cual se considera equivale al 1.5\% del peso total de una persona, en este caso el paciente pesa $11 \mathrm{Kg}$, por lo que el pie equivaldría a $0.165 \mathrm{Kg}$.

Considerando que el pie actúa en forma de palanca con respecto a la tibia, se consideran el peso del pie como una masa puntual en el centro del pie, la cual se ubica a $2.5 \mathrm{~cm}$ del eje de la tibia, genera un par torsor de $0.6 \mathrm{~N} / \mathrm{m}$ sobre el implante macho (Figura 3).

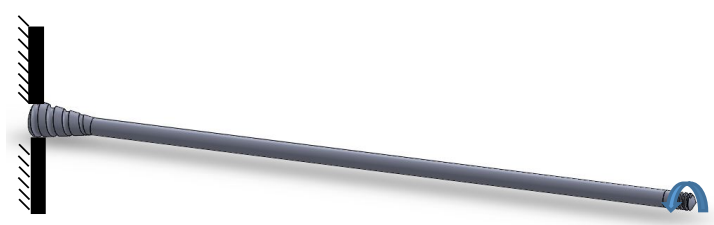

FIGURA 3. Condiciones de frontera en prueba de torsión.

De igual manera al caso anterior se realizó un mallado libre en los dos implantes del dispositivo intramedular. A continuación, en la Tabla 2 se observan los elementos y nodos generados para los análisis.

TABLA 2. Número de elementos y nodos generados en análisis de torsión.

\begin{tabular}{|c|c|c|}
\hline $\begin{array}{c}\text { Dispositivo } \\
\text { para analizar }\end{array}$ & $\begin{array}{c}\text { No. de } \\
\text { elementos }\end{array}$ & $\begin{array}{c}\text { No. de } \\
\text { nodos }\end{array}$ \\
\hline Hexagonal & 10649 & 20246 \\
\hline $\begin{array}{c}\text { Circular } \\
\text { con pestaña }\end{array}$ & 12962 & 24675 \\
\hline $\begin{array}{c}\text { Semicírculo } \\
\text { 3/4 de caña }\end{array}$ & 10819 & 21096 \\
\hline
\end{tabular}

\section{Análisis numérico en ensamble rosca-epífisis}

Se realizaron análisis numéricos considerando un ensamble hueso-implante. Para este se obtuvieron modelos tridimensionales de las epífisis de los huesos del paciente de 6 años con OI. Esto se llevó a cabo 
mediante la realización de una tomografía axial computarizada (TAC) a un paciente con OI tipo III. El estudio tomográfico entrega una serie de imágenes en un formato denominado DICOM. Las imágenes fueron procesadas mediante el programa de cómputo ScanIP ${ }^{\circledR}$, el cual permite realizar modelos virtuales del hueso. Posteriormente, con el apoyo del programa CAD Solidworks ${ }^{\circledR}$ se corrigió la superficie del modelo para convertirlo a un sólido exportable a un programa de cómputo de análisis mediante elemento finito.

Diferentes modelos de rosca son empleados para establecer la interacción que existe en la fijación hueso-implante. La Figura 4 muestra el modelado de la epífisis distal de la tibia del paciente con OI.

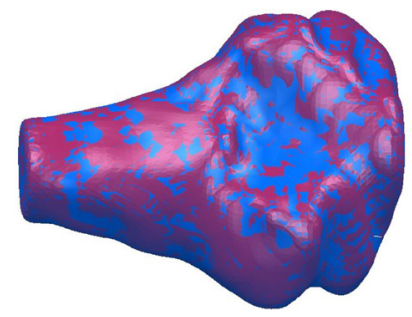

FIGURA 4. Modelo tridimensional de una epífisis distal de tibia.

Se realizó la parametrización de los clavos telescópicos considerando las roscas empleadas actualmente para el tratamiento de la OI. Adicionalmente, se efectuaron rediseños de los clavos modificando el perfil de la rosca, con la finalidad de conocer los esfuerzos generados en el tornillo, así como en el hueso del paciente (Figura 5 y Figura 6).

Se trasladaron los modelos CAD de la tibia al software SolidWorks ${ }^{\circledR}$, con la finalidad de generar ensambles entre el implante macho del clavo telescópico y la epífisis, mostrado el ensamble en la Figura 7.

Para el análisis se introdujo al programa de cómputo, las propiedades mecánicas del hueso con OI, las cuales, un coeficiente de Poisson dentro de la literatura se

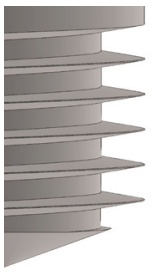

a)

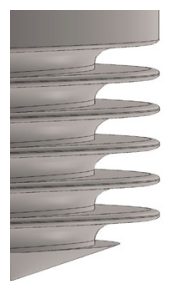

b)

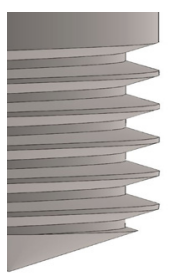

c)
FIGURA 5. Modelos de rosca para epífisis distal. a) Rosca original. b) Rosca Cortical. c) Rosca ACME.

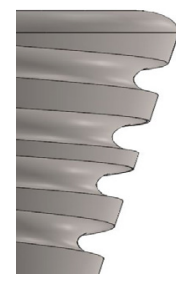

a)

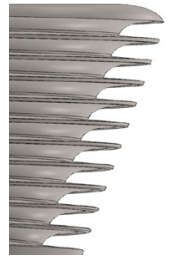

b)

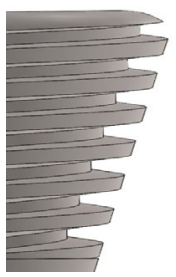

c)
FIGURA 6. Modelos de rosca para epífisis proximal. a) Rosca original. b) Rosca Cortical. c) Rosca ACME.
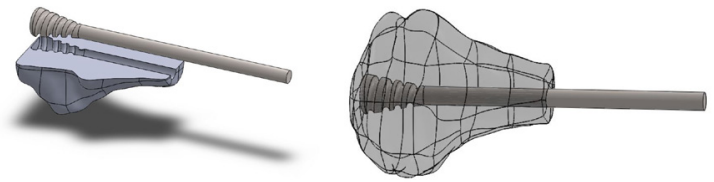

FIGURA 7. Modelo de ensamblaje de epífisis distal con implante hembra. indica que posee un módulo de Young con valores cercanos a 12 GPa de $0.24 \pm 0.08$ y un límite elástico de 49 $\mathrm{MPa}$, mientras que al dispositivo intramedular se le asignaron las propiedades mecánicas del Acero Inoxidable 316L el cual cuenta con un módulo de Young de $200 \mathrm{GPa}$, un coeficiente de Poisson de 0.268 y un límite elástico de $170 \mathrm{MPa}$.

Dentro de la literatura, se indica que pacientes con OI tipo III, durante su infancia, el crecimiento más notable que sufren llega a ser entre 10 y $12 \mathrm{~cm}$ de su estatura total, la cual representa en la tibia un crecimiento promedio de entre 3 y $4.5 \mathrm{~cm}$ [10]. Sin embargo, como se explicó anteriormente, estos dispositivos, al ser telescópicos se van alargando al mismo ritmo que el hueso, por lo que la reacción generada en la rosca es 
mínima. Las condiciones de frontera empleadas para este caso de estudio consistieron en colocar una fijación en las epífisis, al mismo tiempo que se ejercía una fuerza de tensión de $50 \mathrm{~N}$ sobre el dispositivo, la cual será la misma en todos los análisis para poder realizar las comparaciones de los esfuerzos en la rosca,

En la siguiente Tabla 3, se muestra la cantidad de elementos y nodos generados mediante el mallado libre para el análisis de la rosca y la epífisis.

TABLA 3. Número de elementos y nodos utilizados durante el análisis del ensamble rosca-epífisis.

\begin{tabular}{|c|c|c|}
\hline $\begin{array}{c}\text { Dispositivo } \\
\text { para analizar }\end{array}$ & $\begin{array}{c}\text { No. de } \\
\text { elementos }\end{array}$ & $\begin{array}{c}\text { No. de } \\
\text { nodos }\end{array}$ \\
\hline Fassier-Duval ${ }^{\circ}$ & 11459 & 17859 \\
\hline Rosca Cortical & 17454 & 27931 \\
\hline Rosca ACME & 12571 & 19551 \\
\hline
\end{tabular}

\section{Análisis numérico en ensamble hueso-implante}

Finalmente, en los últimos dos casos de estudio se procedió a realizar un análisis considerando un ensamble total del hueso y el dispositivo mostrado en la Figura 8, el cual fue sometido a cargas de flexión, considerando al hueso como una viga en cantiléver y simulando los movimientos de desplazamiento de un niño pequeño con los implantes colocados.

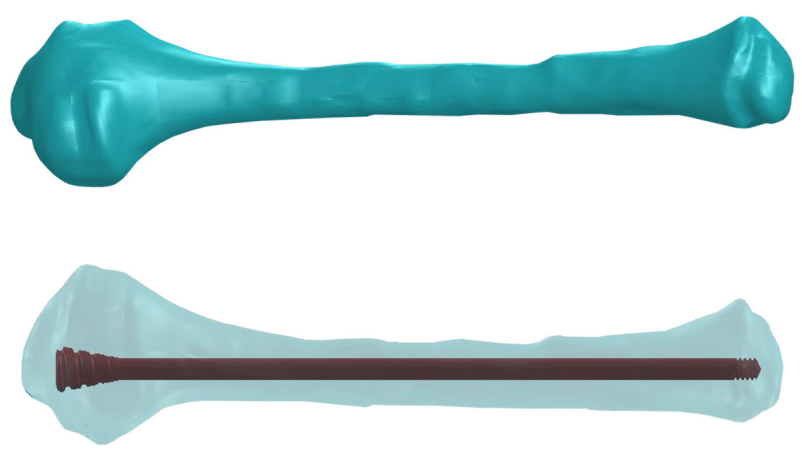

FIGURA 8. Modelo del ensamblaje y condiciones de frontera.
Para estos análisis el mallado libre para el hueso sin implante arrojo una cantidad de elementos de $8763 \mathrm{y}$ de 13898 nodos, mientras que para el ensamble entre hueso-implante género 20068 elementos y un total de 32623 nodos.

\section{RESULTADOS Y DISCUSIÓN}

Los resultados muestran esfuerzos, desplazamientos y deformaciones unitarias de los casos de estudio establecidos; de la misma manera, se recopilaron los datos en la Tabla 4, la Tabla 5, la Tabla 6, la Tabla 7 y la Tabla 8 con la finalidad de comparar el cambio geométrico del alma del implante macho y el anclaje en el ensamble del sistema.

Los resultados de la Tabla 1 y la Tabla 2 muestran los resultados de los primeros 7 casos de estudios correspondientes al análisis de flexión y torsión de los implantes con la modificación geométrica en el alma del dispositivo.

TABLA 4. Resultados de análisis numérico en 4 puntos de flexión.

\begin{tabular}{|l|c|c|c|}
\hline Clavo & $\begin{array}{c}\text { Esfuerzos } \\
\sigma \\
(\mathrm{MPa})\end{array}$ & $\begin{array}{c}\text { Desplazamientos } \\
\delta \\
(\mathrm{mm})\end{array}$ & $\begin{array}{c}\text { Deformaciones } \\
\text { Unitarias } \\
\varepsilon\end{array}$ \\
\hline Fassier-Duval $\mathbb{8}$ & 433.32 & 0.63742 & 0.003382 \\
\hline Hexagonal & 373.72 & 0.63196 & 0.00103 \\
\hline $\begin{array}{l}\text { Circular } \\
\text { con pestaña }\end{array}$ & 448.88 & 0.55171 & 0.0008546 \\
\hline $\begin{array}{l}\text { Semicírculo } \\
\text { 3/4 de caña }\end{array}$ & 338.844 & 0.5663 & 0.001218 \\
\hline
\end{tabular}

TABLA 5. Resultados de torsión en dispositivos intramedulares.

\begin{tabular}{|l|c|c|c|}
\hline Clavo & $\begin{array}{c}\text { Esfuerzos } \\
\sigma \\
(\mathrm{MPa})\end{array}$ & $\begin{array}{c}\text { Desplazamientos } \\
\delta \\
(\mathrm{mm})\end{array}$ & $\begin{array}{c}\text { Deformaciones } \\
\text { Unitarias } \\
\varepsilon\end{array}$ \\
\hline Hexagonal & 973.47 & 0.2321 & 0.003111 \\
\hline $\begin{array}{l}\text { Circular } \\
\text { con pestaña }\end{array}$ & 1289.941 & 0.1731 & 0.004584 \\
\hline $\begin{array}{l}\text { Semicírculo } \\
\text { 3/4 de caña }\end{array}$ & 575.76 & 0.19966 & 0.001648 \\
\hline
\end{tabular}


A continuación, se observan los resultados de los análisis ejecutados a las roscas propuestas y el modelo tridimensional de un hueso con propiedades de OI. La Tabla 6 muestra los resultados para el caso de la epífisis distal, donde es la rosca del implante macho la que interactúa con esta y la Tabla 7 la interacción entre implante hembra y epífisis proximal.

TABLA 6. Resultados en epífisis distal.

\begin{tabular}{|l|c|c|c|}
\hline Tipo de rosca & $\begin{array}{c}\text { Esfuerzos } \\
\sigma \\
(\mathrm{MPa})\end{array}$ & $\begin{array}{c}\text { Desplazamientos } \\
\delta \\
(\mathrm{mm})\end{array}$ & $\begin{array}{c}\text { Deformaciones } \\
\text { Unitarias } \\
\varepsilon\end{array}$ \\
\hline Fassier-Duval ${ }^{\circledR}$ & 23.425 & 0.00203 & 0.000394 \\
\hline Cortical & 42.579 & 0.002157 & 0.00293 \\
\hline ACME & 18.8 & 0.00204 & 0.000495 \\
\hline
\end{tabular}

TABLA 7. Resultados en epífisis proximal.

\begin{tabular}{|l|c|c|c|}
\hline Tipo de rosca & $\begin{array}{c}\text { Esfuerzos } \\
\sigma \\
(\mathrm{MPa})\end{array}$ & $\begin{array}{c}\text { Desplazamientos } \\
\delta \\
(\mathrm{mm})\end{array}$ & $\begin{array}{c}\text { Deformaciones } \\
\text { Unitarias } \\
\varepsilon\end{array}$ \\
\hline Fassier-Duval $\mathbb{}$ & 16.139 & 0.00217 & 0.000348 \\
\hline ACME & 30.565 & 0.002094 & 0.001355 \\
\hline Cortical & 55.464 & 0.00228 & 0.001411 \\
\hline
\end{tabular}

Asimismo, en la Figura 9 se puede observar un diagrama que permite observar las zonas que sufren un mayor desplazamiento al momento de ejercer una fuerza axial a lo largo de la epífisis.

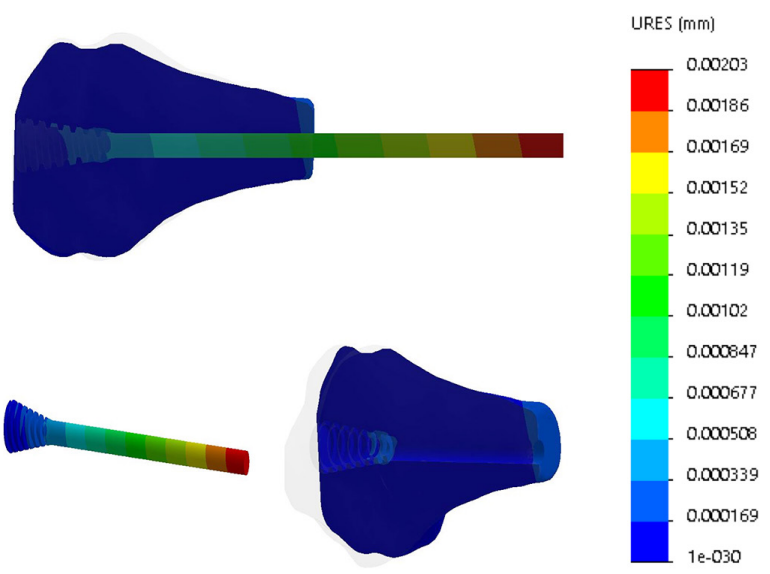

FIGURA 9. Análisis del desplazamiento de las roscas.
Finalmente, se observó que, al colocar el implante dentro del hueso se evita la flexión del tejido óseo, como se observa en la Figura 10, los esfuerzos se concentran en el dispositivo intramedular, permitiendo mantener el hueso recto y así, prevenir fracturas.
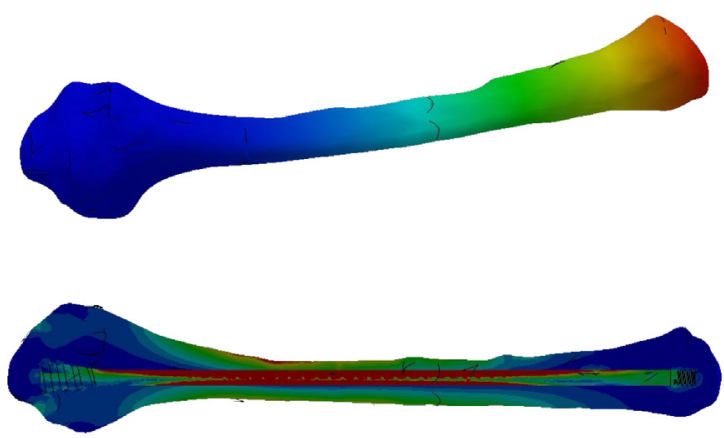

FIGURA 10. Deformaciones unitarias y esfuerzos de Von Mises en hueso con OI con implante.

Los esfuerzos encontrados en el hueso con el dispositivo intramedular son menores comparándolos con el hueso sin implante, lo siguiente se puede observar en la Tabla 8, donde se exhibe el esfuerzo máximo encontrado en la superficie del hueso en ambos análisis, mientras que el generado en las epífisis del hueso aumenta debido al anclaje generado por el dispositivo.

\section{TABLA 8. Esfuerzos del análisis} en el ensamble hueso-implante.

\begin{tabular}{|l|l|l|l|}
\hline & $\begin{array}{l}\text { Esfuerzo } \\
\text { sobre la superficie } \\
\text { del hueso } \\
\text { MPa }\end{array}$ & $\begin{array}{l}\text { Esfuerzo } \\
\text { en epífisis } \\
\text { distal } \\
\text { MPa }\end{array}$ & $\begin{array}{l}\text { Esfuerzo } \\
\text { en epifisis } \\
\text { proximal } \\
\text { MPa }\end{array}$ \\
\hline $\begin{array}{l}\text { Hueso } \\
\text { sin implante }\end{array}$ & 402.06 & 14.92 & 4.24 \\
\hline $\begin{array}{l}\text { Hueso } \\
\text { con implante }\end{array}$ & 260.22 & 67.60 & 57.07 \\
\hline
\end{tabular}

Lo anterior radica en las propiedades mecánicas de los elementos implicados en el ensamblaje, como se observa en la Figura 10, el acero inoxidable 316 L, posee una mayor resistencia a deformarse, por lo que puede decirse que, en un análisis a flexión, el hueso únicamente se deformará en el punto que el implante sufra una deformación. 


\section{CONCLUSIONES}

Con los datos obtenidos se puede observar fácilmente que la propuesta con mayor rigidez a la torsión es el clavo semicírculo 3/4 de caña. Mientras que el clavo que sufre una mayor deformación es el circular con pestaña, por lo que se puede concluir que, si bien el clavo circular con pestaña ofrece una mayor rigidez a la flexión, es fácilmente deformable a la torsión.

Con base en los resultados numéricos obtenidos de las propuestas en flexión y torsión, se puede concluir que, dentro de las geometrías propuestas, la de sección semicírculo 3/4 de caña es la que presenta una menor concentración de esfuerzos a la flexión, así como una menor deformación, bajo esta hipótesis este sería el rediseño con el que se pueden atacar las debilidades existentes dentro del clavo comercial que actualmente se utiliza para el tratamiento de la OI.

De acuerdo con los resultados de los análisis realizados a las roscas se puede observar que la ACME presenta una menor cantidad de esfuerzos en la zona de la epífisis distal, con lo que se puede argumentar que este tipo de rosca al no sufrir concentraciones de esfuerzos no daña la integridad del hueso, por consiguiente, la sujeción del implante con el hueso es mayor que con la rosca con la que actualmente cuneta el dispositivo.

En el caso de la epífisis proximal, el tipo de rosca que presenta menor concentración de esfuerzos es la que actualmente se encuentra en el dispositivo intramedu- lar comercial, si bien los esfuerzos generados entre la rosca ACME y el clavo comercial crecen aproximadamente el doble, en esta sección debido a la forma cónica del extremo roscado proporciona una mayor sujeción entre el clavo y la epífisis, en cuanto al análisis tibial se refiere.

Finalmente, se observa que el clavo telescópico, le brinda una mejor resistencia ante los desplazamientos, asimismo, se encarga de actuar de concentrador de los esfuerzos que se generan durante la aplicación de la carga y de esta manera reducir los esfuerzos en el hueso.

Con los resultados obtenidos, se continuará la investigación realizando la manufactura del dispositivo intramedular con la finalidad de realizar pruebas experimentales ex vivo planteadas en este trabajo, en tejido óseo de carnero, esto debido a la similitud del canal intramedular con el tejido óseo humano con condiciones de OI.

\section{AGRADECIMIENTOS}

Los autores de este trabajo agradecen al Instituto Politécnico Nacional, Consejo Nacional de Ciencia y Tecnología, Asociación Angelitos de Cristal I.A.P., y a la Universidad Politécnica de Madrid por el apoyo brindado en la realización del presente trabajo. Los autores también reconocen el apoyo parcial del proyecto 20181141, así como la subvención EDI, todo proporcionado por SIP / IPN. 


\section{REFERENCIAS}

[1] Shapiro, J. R., Byers, P. H., Glorieux, F. H. y Sponseller, P.D., Osteogenesis Imperfecta: A Translational Approach to Brittle Bone Disease, Academic Press, United States of America, pp. 5-14, 2014. DOI:10.1016/C2011-0-07790-6

[2] Rommens, P.M., Hessmann, M. H. y Editores, Intramedullary Nailing. A Comprehensive Guide, Springer, pp. 565-572, 2015. DOI:10.1007/978-1-4471-6612-2

[3] Rohrbach, M., Giunta, C., Recessive osteogenesis imperfecta: clinical, radiological, and molecular findings, American Journal of Medical Genetics Part C, Seminars in Medical Genetics, Vol. 160, 2012. DOI: 10.1002/ajmg.c.31334

[4] Shapiro, J. R., Byers, P. H., Glorieux, F. H. y Sponseller, P.D., Osteogenesis Imperfecta: A Translational Approach to Brittle Bone Disease, Academic Press, United States of America, pp. 421-426, 2014. DOI:10.1016/C2011-0-07790-6

[5] Sofield, H. A., Millar, A., Fragmentation, realignment, and intramedullary rod fixation of deformities of the long bones in children: a ten year appraisal, The Journal of Bone \& Joint Surgery, Vol. 41, 1959. DOI: 10.2106/00004623-195941080-00001

[6] Porat, S., Heller, E., Seidman DS, Meyer, S., Functional results of operation in osteogenesis imperfect: elongating and nonelongating rods, Journal of Pediatric Orthopaedics, Vol. 11, 1991. DOI: 10.1097/01241398-199103000-00012
[7] Rommens, P.M., Hessmann, M. H. y Editores, Intramedullary Nailing. A Comprehensive Guide, Springer, pp. 565-572, 2015. DOl:10.1007/978-1-4471-6612-2

[8] Zaifeng F., Peter A. S., Gerald F. H., Frank R. Y Ruta B., Comparison of Nanoindentation Measurements Between Osteogenesis Imperfecta Type III and Type IV and Between Different Anatomic Locations (Femur/Tibia versus Iliac Crest), Connective Tissue Research, Vol. 48, pp. 70-75, 2007. DOI: $10.1080 / 03008200601090949$

[9] Huiskes, R., Mow, Van C., Basic Orthopaedic Biomechanics \& Mechano-biology, Lippincott Williams \& Wilkins, 3rd Edition, United States of America, pp. 29-91, 2005. DOI: $10.1186 / 1475-925 X-4-28$

[10] Enagonia, E., Nutrition in Osteogenesis Imperfecta, Osteogenesis Imperfecta: A Translational Approach to Brittle Bone Disease, Academic Press, United States of America, pp. 371-379, 2014. DOI:10.1016/C2011-0-07790-6 